\title{
The Spinning Period of a Free Electron and the Periods of Spin and Orbital Motions of Electron in Atomic States
}

\author{
Ziya Saglam', Mesude Saglam², S. Burcin Bayram³, Tim Horton ${ }^{3}$ \\ ${ }^{1}$ Department of Physics, Aksaray University, Aksaray, Turkey \\ ${ }^{2}$ Department of Physics, Ankara University, Ankara, Turkey \\ ${ }^{3}$ Department of Physics, Miami University, Oxford, Ohio, USA \\ Email: zsaglam@aksaray.edu.tr, saglam@science.ankara.edu.tr, bayramsb@miamioh.edu, \\ hortonts@miamioh.edu
}

Received 30 November 2015; accepted 26 December 2015; published 29 December 2015

Copyright (C 2015 by authors and Scientific Research Publishing Inc.

This work is licensed under the Creative Commons Attribution International License (CC BY).

http://creativecommons.org/licenses/by/4.0/

(c) (i) Open Access

\section{Abstract}

The spinning period for a free electron and the periods of spin and orbital motion of the electron in an atomic state have been calculated. We have shown that for a free electron the spinning period is: $\left(T_{s}\right)_{\text {free }}=1.9 \times 10^{-20} \mathrm{~s}$. But in the atomic case we show that, both the spin and the orbital periods depend on the quantum numbers $n, m_{l}, m_{s}$ and the effective Landé-g factor, $g^{*}$ which is a function of the quantum number $l$ of the atomic state $\left|n, l, m_{l}, m_{s}\right\rangle$ given in Dirac notation. We have also calculated these periods for the ground state and some excited states-hydrogen and hydrogen-like atoms. For atomic states the approximate values of spinning period are $\left(T_{s}\right)_{\text {atomic }} \cong 10^{-21} \mathrm{~s}$ and the related orbital periods are: $\left(T_{0}\right)_{\text {atomic }}=\left(10^{-16}-10^{-15}\right) \mathrm{s}$. Therefore atto-second processes which are related to the pulse of $10^{-18} \mathrm{~s}$ will filter the orbital motion of the electron but will be long enough to detect the details of the spin motion, such as flip-flops.

\section{Keywords}

Electron Spin, Landé-g Factor, Magnetic Top Model, Spinning Period, Atto-Seconds Processes

\section{Introduction}

To calculate the periods of spin and orbital motions of an electron in an atomic state $\left|n, l, m_{l}, m_{s}\right\rangle$ in Dirac re- 
presentation, we consider the total magnetic moment of an electron in the presence of a magnetic field in the $z$ direction. The $z$-component of the total magnetic moment of electron is given by [1]

$$
\mu_{\text {total }}=\mu_{B}\left(m_{l}+g^{*} m_{s}\right)=\mu_{B} m_{l}+\mu_{B} g^{*} m_{s}=\mu_{l}+\mu_{s}
$$

where $\mu_{B}$ is the Bohr magneton, which is given by $\hbar e / 2 m c$ and $g^{*}$ is the effective Landé-g factor which takes the values $g^{*}=l+1=1,2,3,4,5$ depending on the values of the outermost electrons and $l=0,1,2,3,4$ (corresponding to the so called $s, p, d, f$ states respectively).

To calculate the spin period of an electron, we will use the magnetic top model which was first introduced by Barut et al. [2]. For calculating the period of the orbital motion we will use the current loop model [3]-[7].

\section{Period of the Spinning Motion of Electron}

From Equation (1) the $z$-component of magnetic moment associated with the spinning motion is:

$$
\mu_{s}=\mu_{B} g^{*} m_{s}= \pm \frac{g^{*}}{2} \mu_{B}= \pm \frac{g^{*}}{2}\left(\frac{e \hbar}{2 m c}\right)
$$

To proceed further, we calculate the intrinsic magnetic moment of electron with a semiclassical, magnetic top model which was first introduced by Barut et al. [2].

In the magnetic top model, the spin angular momentum of electron is produced by the spinning of the electronic charge (-e) which is assumed to be uniformly distributed inside a sphere of a radius $R$. We denote the spin angular frequency of the rotating charged sphere by $\omega_{s}$, then the magnitude of the magnetic moment of this sphere can be calculated(Appendix I) to be

$$
\mu_{\text {sphere }}=\frac{e \omega_{s} R^{2}}{5 c}
$$

In the presence of the magnetic field $\boldsymbol{B}=B \hat{z}$, the $z$-component of the magnetic moment of the spinning sphere becomes:

$$
\mu_{z}= \pm \frac{e \omega_{s} R^{2}}{5 c}
$$

If we compare Equation (2) and Equation (4) we can write:

$$
\begin{aligned}
& \omega_{s}=\frac{5 g^{*} \hbar}{4 m R^{2}} \\
& T_{s}=\frac{8 \pi R^{2} m}{5 g^{*} \hbar}
\end{aligned}
$$

where $T_{s}$ is the spinning period.

Let us consider the equatorial velocity of this spinning sphere, $v=R \omega_{s}$. A simple relativistic argument shows that $v=c$. Therefore from Equation (5a) we can write:

$$
v \equiv R \omega_{s}=\frac{5 g^{*} \hbar}{4 m R}=c
$$

Which defines the radius of electron as below:

$$
R=\frac{5 g^{*} \hbar}{4 m c}
$$

For a free electron $g^{*}=2$ substituting other related variables in Equation (7) gives us the radius of a free electron, $R_{\text {free }}$ :

$$
R_{\text {free }}=9 \times 10^{-11} \mathrm{~cm}
$$

Substitution of Equation (8) in Equation (5b) gives us the spinning period for a free electron:

$$
\left(T_{s}\right)_{\text {free }}=1.9 \times 10^{-20} \mathrm{~s}
$$


which is in good agreement with the semiclassical calculation of Olszewski [8].

For an electron in an atom, we cannot calculate the radius directly from Equation (7), because we need to know the effective values of $g^{*}$. For the same reason we must take the effective values of $g^{*}$ in Equation (5b) which gives us $\left(T_{s}\right)_{\left(n, l, m_{l}, m_{s}\right)}$ for the state $\left|n, l, m_{l}, m_{s}\right\rangle$.

In the following section we find an expression for the period of orbital motion, $\left(T_{0}\right)_{\left(n, l, m_{l}, m_{s}\right)}$ for the outermost electron in hydrogen and hydrogen-like atoms: which is given by Equation (14):

$$
\left(T_{0}\right)_{\left(n, l, m_{j}, m_{s}\right)}=\frac{2 \pi r_{n}^{2} m}{\hbar\left|m_{l}+g^{*} m_{s}\right|}
$$

When we take the ratio of the periods given in Equation (5b) and Equation (14), we find:

$$
\frac{\left(T_{s}\right)_{\left(n, l, m_{l}, m_{s}\right)}}{\left(T_{o}\right)_{\left(n, l, m_{l}, m_{s}\right)}}=\frac{4\left|m_{l}+g^{*} m_{s}\right|}{5 g^{*}} \frac{R^{2}}{r_{n}^{2}}
$$

Substituting $R=\frac{5 g^{*} \hbar}{4 m c}$ (from Equation (7)) and $r_{n}=n^{2} a_{0}$ with $a_{0}=\frac{\hbar^{2}}{m e^{2}}$ we get:

$$
\left(T_{s}\right)_{\left(n, l, m_{l}, m_{s}\right)}=\frac{\left|m_{l}+g^{*} m_{s}\right| e^{4}\left(g^{*}\right)}{4 n^{4} \hbar^{2} c^{2}}\left(T_{o}\right)_{\left(n, l, m_{l}, m_{s}\right)}
$$

It is known that when there is no quantum entanglement, for a free electron, the Landé-g factor is equal to 2. For an electron in an atom the Landé-g factor is given by:

$$
g=1+\frac{J(J+1)+S(S+1)-L(L+1)}{2 J(J+1)}
$$

which varies is in range of $g_{\min }=1<g<2=g_{\max }$. Recently, Saglam et al. [1] showed that because of the quantum entanglements in an atom the Landé-g factor is replaced by the effective g-factor, $g^{*}$ which takes the values $g^{*}=l+1=1,2,3,4,5$ depending on $l=0,1,2,3,4$ (corresponding to the so called $s, p, d, f$ states respectively) values of the outermost electrons together with the unfilled shells respectively. So the maximum values of the effective Landé-g factor, $g^{*}$ can be as high as 5. Therefore $g_{\min }^{*}=1<g^{*}<g_{\max }^{*}=5$.

If we calculate the effective g-factor, $g^{*}$ for the ground state hydrogen atom, $|1,0,0,1 / 2\rangle$, we found that $g^{*}=1$. For this value we calculate the period of ground state orbit and find: $\left(T_{o}\right)_{(1,0,0,1 / 2)}=2.6 \times 10^{-16} \mathrm{~s}$. Substituting this value and $n=1, m_{l}=0, m_{s}=1 / 2, g^{*}=1$ and other related parameters in Equation (11), we find the spinning period of electron in $|1,0,0,1 / 2\rangle$ state, $\left(T_{s}\right)_{(1,0,0,1 / 2)} \cong 1.48 \times 10^{-21} \mathrm{~s}$. We give the values of $\left(T_{s}\right)_{\left(n, l, m_{j}, m_{s}\right)}$ and $\left(T_{0}\right)_{\left(n, l, m_{j}, m_{s}\right)}$ for the states: $|1,0,0,1 / 2\rangle,|2,1,0,1 / 2\rangle,|3,2,2,1 / 2\rangle,|4,3,1,1 / 2\rangle$ and $|4,3,2,1 / 2\rangle$ in Table

\begin{tabular}{|c|c|}
\hline Spinning periods & Periods of orbital motion \\
\hline$\left(T_{s}\right)_{(1,0,0,1 / 2)} \cong 1.48 \times 10^{-21} \mathrm{~s}$ & $\left(T_{0}\right)_{(1,0,0,0,1 / 2)}=2.6 \times 10^{-16} \mathrm{~s}$ \\
\hline$\left(T_{s}\right)_{(2,1,0,01 / 2)} \cong 7.66 \times 10^{-21} \mathrm{~s}$ & $\left(T_{0}\right)_{(2,1,0,0 / 2)}=2.08 \times 10^{-15} \mathrm{~s}$ \\
\hline$\left(T_{s}\right)_{(3,2,2,1 / 2)} \cong 1.77 \times 10^{-21} \mathrm{~s}$ & $\left(T_{0}\right)_{(3,2,2,1 / 2)}=6.4 \times 10^{-16} \mathrm{~s}$ \\
\hline$\left(T_{s}\right)_{(4,3,1,1,2)} \cong 1.59 \times 10^{-21} \mathrm{~s}$ & $\left(T_{0}\right)_{(4,3,1,1,1 / 2)}=2.3 \times 10^{-15} \mathrm{~s}$ \\
\hline$\left(T_{s}\right)_{(4,3,2,2,1 / 2)} \cong 1.79 \times 10^{-21} \mathrm{~s}$ & $\left(T_{0}\right)_{(4,3,2,2,1 / 2)}=1.1 \times 10^{-15} \mathrm{~s}$ \\
\hline
\end{tabular}
1.

\section{Period of the Orbital Motion of Electron}

From Equation (1) the z-component of the total magnetic moment is: 


$$
\mu_{j}=\mu_{\text {total }}=\mu_{B}\left(m_{l}+g^{*} m_{s}\right)=\mu_{B} m_{l}+\mu_{B} g^{*} m_{s}=\mu_{l}+\mu_{s}
$$

where $m_{l}=0, \pm 1, \cdots, \pm l, m_{s}= \pm 1 / 2, \quad \mu_{B}=\frac{e \hbar}{2 m c}$ and $g^{*}=1,2,3,4,5$.

Now we find another expression for $\mu_{\text {total }}$ in the current loop model [2]: we assume that the magnetic moment associated with the orbital motion of electron is produced by the fictitious point charge (-e) rotating in a circular orbit with the angular frequency $\omega_{j}=2 \pi / T_{j}$ and the radius $r_{n}$ in $x$-y plane. In this model the $z$-component of the magnetic moment will be

$$
\mu_{\text {total }}=\frac{I A}{c}=\frac{e \omega_{j} \pi r_{n}^{2}}{2 \pi c}=\frac{\pi e r_{n}^{2}}{c T_{\text {total }}}
$$

If we compare Equation (12) and Equation (13) we write:

$$
\left(T_{0}\right)_{\left(n, l, m_{j}, m_{s}\right)}=\frac{2 \pi r_{n}^{2} m}{\hbar\left|m_{l}+g^{*} m_{s}\right|}
$$

where we replace $T_{\text {total }}$ by $\left(T_{0}\right)_{\left(n, l, m_{j}, m_{s}\right)}$; here the subscript (0) stands for orbital motion.

Now we can find the values of $\left(T_{0}\right)_{\left(n, l, m_{j}, m_{s}\right)}$ for hydrogen and hydrogen-like atoms: especially for $\left|n, n-1, m_{l}, m_{s}\right\rangle$ states we can put $r_{n}=n^{2} a_{0}$ where $a_{0}=\frac{\hbar^{2}}{m e^{2}}$.

With these replacements Equation (14) becomes:

$$
\left(T_{0}\right)_{\left(n, l, m_{l}, m_{s}\right)}=\frac{2 \pi r_{n}^{2} m}{\hbar\left|m_{l}+g^{*} m_{s}\right|}=\frac{2 \pi \hbar^{3} n^{4}}{m e^{4}\left|m_{l}+g^{*} m_{s}\right|}
$$

We note that the quantum number $(l)$ gets involved through the effective Lande-g factor, $g^{*}$ which takes the values $g^{*}=l+1$.

For example, for the ground state of hydrogen atom $|1,0,0,1 / 2\rangle$, substituting $g^{*}=1$ and the other related parameter in Equation (15), we find:

$$
\left(T_{0}\right)_{(1,0,0,1 / 2)}=2.6 \times 10^{-16} \mathrm{~s}
$$

Similarly for the state $|2,1,0,1 / 2\rangle$ the corresponding period is:

$$
\left(T_{0}\right)_{(2,1,0,1 / 2)}=2.08 \times 10^{-15} \mathrm{~s}
$$

where we put: $n=2, l=1, g^{*}=l+1=2$ and $m_{l}=0$ in Equation (15).

\section{Acknowledgments}

Authors from Miami University acknowledge financial support from the National Science Foundation (Grant No. NSF-PHY-1309571).

\section{References}

[1] Saglam, Z., Bayram, S.B. and Saglam, M. (2010) Journal of Modern Physics, 1, 399-404. http://dx.doi.org/10.4236/jmp.2010.16057

[2] Barut, O.A., Bozic, M. and Maric, Z. (1992) Annals of Physics, 214, 53-83. http://dx.doi.org/10.1016/0003-4916(92)90061-P

[3] Saglam, M. and Sahin, G. (2009) Journal of Modern Physics, B, 23, 4977-4985.

[4] Saglam, M., Saglam, Z., Boyacioglu, B. and Wan, K.K. (2007) Journal of Russian Laser Research, 28, 267-271. http://dx.doi.org/10.1007/s10946-007-0015-6

[5] Saglam, Z. and Boyacioglu, B. (2007) Journal of Russian Laser Research, 28, 142-147. http://dx.doi.org/10.1007/s10946-007-0008-5 
[6] Saglam, M., Boyacioglu, B. and Saglam, Z. (2007) Journal of Russian Laser Research, 28, 377-382. http://dx.doi.org/10.1007/s10946-007-0026-3

[7] Saglam, M., Saglam, Z. and Boyacioglu, B. (2006) Journal of the Old and New Concepts in Physics, 3, 181-192.

[8] Olszewski, S. (2014) Journal of Modern Physics, 5, 2030-2040. http://dx.doi.org/10.4236/jmp.2014.518199

\section{Appendix I: Calculation of the Magnetic Moment of a Spinning Charge, $Q$ Distributed Uniformly inside a Sphere of Radius $R$}

Let us denote the uniform charge density by $\rho_{0}$ which is related to the total charge $Q$ by:

$$
Q=\int_{0}^{Q} \mathrm{~d} Q=\rho_{o} \int_{0}^{R} 4 \pi r^{2} \mathrm{~d} r=\rho_{o}\left(\frac{4 \pi}{3} R^{3}\right)
$$

where $q \equiv \mathrm{d} Q=\rho_{o}\left(4 \pi r^{2} \mathrm{~d} r\right)$ is the charge of the spherical shell with the radius $r$ and thickness $\mathrm{d} r$. First we want to calculate the magnetic moment of this spherical shell with the surface charge density $\left(\sigma=q / 4 \pi r^{2}\right)$. Let us assume that the spinning is about z-axis with the angular frequency, $\omega_{s}$. Let us consider the charge element $\mathrm{d} q$ in the area of the band with the radius $(r \sin \theta)$ and the thickness $(r \mathrm{~d} \theta)$ in spherical coordinates:

$$
\mathrm{d} q=\sigma \mathrm{d} a=\left(\frac{q}{4 \pi r^{2}} 2 \pi r^{2} \sin \theta\right) \mathrm{d} \theta=\left(\frac{q}{2} \sin \theta\right) \mathrm{d} \theta
$$

The current element $\mathrm{d} I$ produced by the rotating band charge with the angular frequency, $\omega_{s}$ will be:

$$
\mathrm{d} I=\frac{\omega_{s}}{2 \pi} \mathrm{d} q=\left(\frac{\omega_{\mathrm{s}} q}{4 \pi} \sin \theta\right) \mathrm{d} \theta
$$

The magnetic moment element of this band current will be:

$$
\mathrm{d} \mu_{\text {band }}=\frac{\mathrm{d} I}{c} \pi(r \sin \theta)^{2}=\left(\frac{\omega_{s} q r^{2}}{4 c} \sin ^{3} \theta\right) \mathrm{d} \theta
$$

Integrating over the spherical shell gives us the magnetic moment of this shell, $\mu_{\text {shell }}$ :

$$
\begin{aligned}
\mu_{\text {shell }} & =\int \mu_{\text {band }}=\int_{0}^{\pi}\left(\frac{\omega_{s} q r^{2}}{4 c} \sin ^{3} \theta\right) \mathrm{d} \theta=\frac{\omega_{s} q r^{2}}{4 c} \int_{0}^{\pi} \sin ^{3} \theta \mathrm{d} \theta \\
& =\frac{\omega_{s} q r^{2}}{2 c} \int_{0}^{\pi / 2}\left(1-\cos ^{2} \theta\right) \sin \theta \mathrm{d} \theta=\frac{\omega_{s} q r^{2}}{2 c} \int_{0}^{1}\left(1-x^{2}\right) \mathrm{d} x=\frac{\omega_{s} q r^{2}}{3 c}
\end{aligned}
$$

If we substitude $q \equiv \mathrm{d} Q=\rho_{o}\left(4 \pi r^{2} \mathrm{~d} r\right)$ in (A-V) and integrate over the spherical volume, we find the total magnetic moment of the sphere of radius $R$ :

$$
\mu_{\text {sphere }}=\int_{0}^{R} \mathrm{~d} \mu_{\text {sphere }}=\int_{0}^{R} \frac{\omega_{s} r^{2}\left(\rho_{o} 4 \pi r^{2} \mathrm{~d} r\right)}{3 c}=\frac{4 \pi \omega_{s} \rho_{o}}{3 c} \int_{0}^{R} r^{4} \mathrm{~d} r=\frac{4 \pi \omega_{s} \rho_{o}}{3 c} \frac{R^{5}}{5}
$$

Substituting (A-I) in (A-VI) we find:

$$
\mu_{\text {sphere }}=\frac{Q \omega_{s} R^{2}}{5 c}
$$

